\title{
Materialien zur Deutung von stipulatio in mittelalterlichen Urkunden.
}

\author{
Von \\ Herrn Professor Dr. Lothar Seuffert \\ in Giessen.
}

Ueber den Sinn der in fränkischen Formeln und Urkunden häufig wiederkehrenden Klausel stipulatione (adstipulatione) subnixa ist eine Einigung der Ansichten noch nicht erzielt.

Die zwei deutschen Schriftsteller, welche zuletzt über den Sinn der Worte sich vernehmen liessen, sind R. Löning (Vertragsbruch, S. 17, Note 28) und Brunner (zur Rechtsgeschichte der römischen und germanischen Urkunde, S. $221 \mathrm{ff.}$ Daselbst ist die frühere Literatur unter Anfuihrung der hauptsächlichen Ansichten verzeichnet). Löning hält die Kilausel für eine blosse Bekräftigungsformel ohne reelle Bedeutung. Nach Brunner soll stipulatio die signa und die Unterschriften der Urkunde bezeichnen, stipulatio subnixa im Texte der Urkunde auf das Signiert- und Unterzeichnetsein verweisen.

Jede Vermehrung des urkundlichen Materials wird zur Klärung der Ansichten beitragen. Eine solche Vermehrung bringt das Urkundenbuch der Stadt Strassburg, erster Band, Urkunden und Stadtrechte bis zum J. 1266, bearbeitet von W. Wiegand (Strassburg 1879).

Darin finden sich neun Urkunden mit der typischen Klausel stipulatione subnixa. Ich stelle die interessierenden Urkundentheile zusammen:

1.

Nr. 14. Sigibald schenkt dem Kloster Weissenburg Güter und Viehheerden etc. $\mathbf{7 7 4}$ Mai 11. 
.... Si quis vero, quod futurum esse non credo, nos ipsi aut aliquis de heredibus nostris hanc pro heredibus meis seu quislibet ulla persona, qui contra hanc paginolam testamenti adhere conaverit aut eam infrangere voluerit, inferat aut partibus fisci auri libram 1, argenti pondus 2 coactus exsolvat et quod repedit evindicare non valeat stipulacione subnixa.

2.

Nr. 16. Bischof Remigius von Strassburg überträgt der Kirche der heil. Maria daselbst seinen ganzen Besitz nebst dem Kloster auf der Insel Eschau etc. 778 März 15. Strassburg.

.... Ego itaque Remigius etsi peccator vilissimus servus servorum dei gratia episcopus Argentinensis urbis sana mente sanoque consilio hoc testamentum [feci], quod ego plena devotione et unita voluntate condidi, quod ego ipse dictavi et manibus meis scripsi et subscripsi adhibitis legitimi numeri testibus, qui a nobis rogati subter subscripserunt et signacula manibus suis roboraverunt. quod testamentum volo ut advicem omnium codicellorum plenissimam obtineat firmitatem, et si causa juris civilis pretorii aliquis tamquam intestatum velit infirmare, et ut id non valeat, dentur testes huic heredi mee sacrosanctae ecclesie etc. efc....

$\ldots \ldots \ldots$ Si quis vero vel quecunque persona quolibet tempore quocunque ingenio contra hoc testamentum... venire aut agere temptaverit aut contrarius esse voluerit aut minuere aut irrumpere conaverit, imprimis iram dei incurrat et ab antistite, qui tunc temporis est, coram deo et sancta Maria excommunicatus existat..... et insuper inferat sanctorum actoribus sacrosancte ecclesie sancte Marie heredi mee una cum sacratissimo fisco auri libras 5, argenti pondera 25 coactus exsolvat, et quod repetit vindicare non valeat, et nihilominus presens pagina testamenti firma stabilitate debeat permanere cum stipulatione subnixa.

Die Urkunde ist wahrscheinlich gefälscht ${ }^{1}$ ). Die Fälschung muss, der Sprache und den gebrauchten Formeln nach zu urtheilen, entweder in die fränkische Zeit fallen oder doch

1) Urkundenbuch, Anmerkungen zu Nr. 16, S. 14. 
nach Mustern aus dieser Zeit gefertigt sein. Man vergleiche die unten folgenden Urkunden Nr. 19 und 21.

3.

Nr. 17. Imma verkauft ihrem Sohne Güter im Elsass. 778 März 21.

Nach Strafgeding heisst es: Et nihilominus sed presens venditio haec omni tempore firma et inviolata permaneat stipulatione subnixa.

4.

Nr. 18. Alderich verkauft dem Mönch Rihbald . . ein Grundstück mit Haus. 780 November 10. Strassburg.

Si quis vero, quod fieri non credo, et cetera (die Abkürzung steht in der Urkunde) stipulacione subnixa.

5.

Nr. 19. Voto schenkt dem Kloster Fulda Güter im Elsass. 788 April 18. Schäffolsheim.

Nach Strafgeding heisst es: sed presens donatio haec omni tempore firma et inviolata permaneat stipulatione subnixa.

6.

Nr. 21. Theothard schenkt für das Seelenheil Adalharts dem Kloster Fulda ein überbautes Grundstück etc. 801 Juni 27. Strassburg.

Im Anschluss an Strafgeding: sed presens donatio haec omni tempore firma et inviolata permaneat stipulatione subnixa.

7.

Nr. 37. Dietbald überträgt der Kirche der heil. Maria in Strassburg Ackerland. 910 Febr. 4. Strassburg.

Nach Strafgeding: Sed hec presens traditio omni tempore firma et stabilis permaneat stipulatione subnixa.

8.

Nr. 38. Wizericus und sein Bruder Azzo schenken der Münsterkirche in Strassburg vierundvierzig Hufen etc. 951 September 26. Strassburg. 
Im Anschluss an Strafindrohung heisst es: sed [sc. haec charta] firma et stabilis permaneat stipulatione subnixa.

9.

Nr. 49. Irimfried schenkt für den Fall seines Todes den an der Kirche der heil. Maria zu Strassburg dienenden Brüdern sein Gut. 965 - 991. Strassburg.

Im Anschluss an Strafandrohung heisst es: et haec translegatio nihilominus stabilis perseveret et fixa stipulatione subnixa.

Ferner kommt stipulatio, adstipulatio (astipulatio), abstipulari (astipulari) vor in den Urkunden:

Nr. 25. Kaiser Lothar bestätigt auf Bitten der Aebtissin Basilla dem Frauenkloster St. Stephan zu Strassburg die Immunität und schenkt demselben zwölf Höfe. 845 Mai 15. Strassburg.

... proinde vero eisdem confirmatis, hortatu et rogatu atque favore voluntario dilectissime conjugis imperatricis auguste Hyrmingardis, collecta utriusque manu, stibulatione firma, donatione legitima tradidimus jam etc. etc.

Nr. 41. Bischof Udo von Strassburg schenkt den an der Kirche der hl. Maria daselbst dienenden Brüdern in der Ortenau und im Elsass gelegene Güter. 961.

.... notum sit igitur, ... qualiter ego Ödo ... predium unum Argentoratensis ecclesie procuratrici gloriosissime dei genetrici Marie ibidemque sibi famulantibus pro remedio animarum parentum meorum nostreque anime salute legitima astipulatione cum viis et inviis, pratis et arvis, cultis et incultis ceterisque utensilibus contradidi.

Nr. 63. Der Strassburger Kanonikus Burchard überträgt in Gemeinschaft mit seinen Brüdern ... dem Strassburger Domstift sein in der Gemarkung Willgottheim gelegenes Gut. 1100 Dezember 29.

- Notum igitur esse cupio .... qualiter ego ... fratresque mei .... Argentinensi ecclesie .... predium nostrum ... tradidimus, astipulatione legitima firmavimus, justis investituris investivimus. 
Nr. 65. Bischof Cuno von Strassburg beurkundet, dass seine Getreuen ... dem Strassburger Domstift Grundbesitz ... geschenkt habell. 1105. Strassburg.

.... notum esse cupimus, ... qualiter quidam laici .... decem mansos .... manutradiderunt, tradendo legitima astipulatione confirmaverunt etc.

Nr. 88. Beurkundung früherer Schenkungen. 11411143.

- . prefatus Cůnradus prepositus omnes ministeriales suos cum prediis, filiis et universis possessionibus suis . . per manum advocati sui beate Marie legali astipulatione delegavit etc.

Nr. 90. Bischof Burchard von Strassburg bestätigt die von seinen Vorgängern Cuno und Gebhard, sowic zu seiner Zeit dem Strassburger Hospital gemachten Schenkungen. 1143. facta sunt vero hec presentibus canonicis ... (folgen die Namen) ... presentibus etiam et astipulantibus bone memorie laicis (folgen die Namen).

- Gelpheradus etiam prefatus eodem die dedicationis ad capellam hospitalis unum hominem nomine Adelbertum et mansum unum ... pro nomine dotis donavit et abstipulando possidendum perpetuo contradidit.

Nr. 100. Bischof Burchard von Strassburg beurkundet, dass Walther, Mönch in Mauersmünster, dem Frauenkloser Sindelsberg zwei Höfe in der Stadt Strassburg u. A. geschenkt habe. 1148.

. . notificamus .... qualiter Waltherus .... per manum Waltheri causidici et Segemundi beate Marie dei genitrici in Sindelesberch famulantibus firma astipulatione tradidit etc. (folgt die Bezeichnung der Güter) ...... ut autem hujus donationis astipula ti o rata sit, instrumentum presens sigillo nostro munire statuimus etc.

Nr. 111. Konrarl Archipresbyter in Strassburg schenkt scin Haus mit allem Geräth und seiner ganzen Habe dem St. Arbogast-Kloster bei Strassburg. 1161 Dezember 24.

.... et quoniam perversitas et molitio mortalium in cunctis precaveri utilis censetur, tam presentibus quam preteritis sanissime consultum est, quatenus nichilominus fidelitati 
aliorum et manibus consentiente prefato Walthero eodem tenore omnia prememorata crederentur, ne machinationibus ecclesia pretaxata jacturam cujuspiam postea injuste patiatur, sed sub tuitione subjectarum personarum largitio hec astipulatione firma perpetualiter facta $a b$ omnibus habeatur. quarum ista sunt nomina etc.

Aus den sämmtlichen bisher angeführten Urkunden ergiebt sich zunächst das sichere negative Resultat, dass stipulatio astipulatio und adstipulatio mit einem obligatorischen Versprechen nichts $\mathrm{zu}$ thun hat. Ueberall kommen die beiden Ausdrücke in Verbindung mit Entäusserungsgeschäften vor, (auch die letztwilligen Vergebungen sind bekanntlich in dieser Zeit Entäusserungsgeschäfte unter Lebenden). Die stipulatio muss also mit der Sicherung solcher Geschäfte zusammenhängen.

Gewinnt schon hiernach die Brunnersche Annahme eine indirekte Stütze, insofern nämlich die Möglichkeit einer Deutung auf obligatorische Versprechungen entfällt, so ist mindestens aus einem Theile der Urkunden die positive Bestätigung ihrer Richtigkeit zu entnehmen. Als direkte und, soviel ich sehe, unwiderlegliche Zeugnisse für Brunners Ansicht dienen die Urkunden Nr. 90 (S. 119) und Nr. 111 (S. 119). Das astipulari der Zeugen in Nr. 90 kann wohl nicht anders als auf die Unterschriften gedeutet werden. Das nachfolgende abstipulando tradere in derselben Urkunde gewinnt, da astipulari und abstipulari wohl in gleichem Sinn gebraucht sind, dadurch auch eine Beziehung auf die Beurkundung. Die astipulatio in Nr. 111 muss von dem Beurkundungsakt verstanden werden.

Sehr nahe liegt die Beziehung der stibulatio in Nr. 25 (S. 118) auf die Beurkundung bzw. auf den entscheidenden Act, die Vollziehung durch Unterschrift.

Die übrigen Urkunden sprechen mindestens nicht gegen Brunners Annahme.

Vom dreizehnten Jahrhundert an wird stipulatio in den Strassburger Urkunden nicht mehr, wie früher, in Verbindung mit Uebergabe von Immobilien sondern in Verbindung mit obligatorischen Versprechen gebraucht.

Ich führe folgende Auszüge an: 
Nr. 176. Bischof Heinrich von Basel sowie der Dekan Konrad und der Scholastikus Heinrich der Baseler Kirche schlichten als vom apost. Stuhl bestelite Richter einen Streit zwischen dem Strassburger Domcapitel und dem Hern von Rappoltstein, Abgaben von Sigolsheimer Gütern betr. 1219 Oktober.

- sic ipsam controversiam nobis presentibus et auctoritate nostra amicabiliter inter eos decisam presenti scripto protestamur: videlicet quod dictus Egilophus et Anselmus et ipsi pro eorum heredibus per stipulationem se singulis annis capitulo Argentinensi proxima septimana ante purificationem sancte Marie tres frischingios claustrales porcinos, quatuor amas vini et dimidiam - quartalia frumenti deinceps obligarunt soluturos.

Nr. 180. Bischof Heinrich von Strassburg schliesst mit dem Strassburger Vogt Anselm einen dessen Lehensverhältniss regelnden Vertrag. 1219.

- idem Anshelmus advocatus corporali prestito juramento promisit, se universa sub omnipotentis anathemate fideliter et efficaciter conservaturum renuntiando exceptione fori, appellationi et legi ,si ob es alienum" et breviter exceptionibus universis super aliquo predictorum sibi vel jure civili vel canonico competentibus, promittens etiam per stipulationem, omne dampnum et expensas universas, quas propter hoc ecclesiam Argentinensem vel capitulum ejusdem ex facto suo vel negligentia subire contigerit, integraliter se refecturum.

Nr. 411. Bischof Heinrich von Strassburg verkauft mit Zustimmung der Bürgerschaft den Franziskanern daselbst ein Grundstück. 1257 Nov. 30.

- ad omnia supradicta tenenda et servanda nos nostrosque successores per stipulationem obligantes. (Vorher geht Versprechen der Haftung im Evictionsfalle: promittentes nos facturos et procuraturos, quod eadem area in toto vel in parte non evincatur ab eisdem etc.)

Nr. 425. Bischof Heinrich von Strassburg und sein Official Nikolaus, Probst von St. Thomas, beurkunden einen Vergleich zwischen der Wittwe und den Kindern Burchard 
Bone's einer- und dem St. Katharinenkloster in Strassburg andererseits über Burchards Hinterlassenschaft. 1258 April 17.

Ego Hiltrudis predicta confiteor omnia suprascripta facta fore et acta per me et cum meo pleno et libero consensu et per stipulationem promitto, me omnia et singula supranotata facturam et procuraturam, renuntians omni juri pro bonis dicti mei quondam mariti et actionibus pro me et meis pueris nobis competentibus et competituris etc.

Nr. 465. Symund von Geroldseck verkauft alle seine Besitzungen zu Wolfisheim dem St. Katharinenkloster zu Strassburg. 1261 April.

omnia suprascripta ego Symundus sepefatus me facturum, procuraturum et completurum promitto per stipulacionem sollempnem et promisi, etc. ... insuper ad majorem securitatem omnium et singulorum premissorum nos Heinricus et Peregrinus fratres, Otto de Marley, Lampertus de Sweinheim ad peticionem dicti domini Symundi promittimus stipulacione interposita data fide sub ypoteca rerum nostrarum nobilium et immobilium, nos procuraturos et effecturos, quod omnia et singula premissa ... fideliter observabuntur rataque et firma perpetuo permanebunt.

Nr. 479. Meister und Rath der Stadt Strassburg beurkunden, dass Bürger Hugo von Saarburg sein zum Steinhof genanntes Haus... dem städtischen Boten Dietrich ... verkauft habe. 1261 Nov. 4.

- Nach Empfangsbekenntniss heisst es vom Verkäufer: promittens pro se suisque heredibus per stipulationem sollempnem, nec litem nec controversiam ipsi Dietrico, suis liberis et heredibus, vel in quem dictus Dietricus domum transtulerit, se moturum, sed dictam domum ab omni homine et etiam ab universitate legittime auctorizare et etiam defensare omneque dampnum et litis expensas..... integraliter resarcire etc. etc.

Nr. 606. Abt Anselm und der Konvent des Klosters Schwarzach verkaufen einen beim Steinburgthor in Strassburg gelegenen Hof für 60 Mark Silber an das Johanniterhospital zu Dorlisheim. 1266 Febr. 5. 
Promittimuseciam per stipulationem sollempnem, nec per nos nec nostros successores litem vel controversian ipsis fratribus, vel eis, in quos predictam curiam transtulerint, nos moturos nec racione minoris precii vel qualibet alia cerciorati, predictam curiam majoris precii esse contra predictam venditionem per nos vel nostros successores aliqua facere vel venire in judicio vel extra.

$\mathrm{Ob}$ die stipulatio in diesen Urkunden des dreizehnten Jahrhunderts auch noch in einer Beziehung auf die signa und Unterschriften der Urkunden steht, wage ich nicht zu entscheiden. Unwahrscheinlich ist es nicht.

\title{
Die Redaktionen des Westgothenrechts durch die Könige Chindasuinth und Reccessuinth.
}

\author{
Von \\ Herrn Stul. jur. R. Schmeltzer \\ in Berlin.
}

Die Entwicklungsgeschichte des westgothischen Gesetzbuches ist im Einzelnen noch sehr wenig aufgehellt. Doch ist durch die eingehenden Vergleichungen der wichtigsten Manuskripte wenigstens so viel gewonnen, dass die Redaktionen der lex Wisigothorum durch die Könige Reccared I, Reccessuinth und Ervig als durch handschriftliche Ueberlieferung sicher festgestellt angesehen werden können.

Bruchstücke der Antiqua Reccared's sind erhalten in dem von Knust 1839 entzifferten Pariser Palimpsest St. Germain 1278. Nach dessen frühem Tode hat sie Bluhme herausgegeben (1847) als ,Reccaredi Wisigothorum regis antiqua legum collectio". Danach war das Gesetzbuch Reccared's in Kapitel und Titel getheilt, aber noch nicht in Bücher.

Eine Gesetzsammlung Reccessuinth's überliefern zwei Handschriften, der Codex Remigianus, Paris 4668, aus dem neunten Jahrhundert, einst dem Kloster S. Remy in Reims gehörigr, und der Codex Vaticanus 1024. Vergl. hierüber 